\title{
A DYNAMIC DATA-DRIVEN APPLICATION SIMULATION MODEL FOR OIL SPILL EMERGENCY DECISION IN PORT WATER AREA
}

\author{
Qingcheng Zeng, Qian Zhang, Zhongzhen Yang \\ School of Transport Management, Dalian Maritime University, China \\ Submitted 10 January 2012; accepted 28 February 2012; \\ first published online 16 October 2013
}

\begin{abstract}
In order to solve the difficulty in simulation and prediction the evolution of oil spill incidents in port water area caused by system complexity and environment variety, a simulation model based on Dynamic Data-Driven Application System (DDDAS) was developed. By assimilating real-time data, simulation model can update its initial condition and solutions, and modify its parameters. Firstly, the framework for DDDAS-based simulation model was designed. Then the method for mapping of real-time data to simulation model, the method for recovery of initial data for DDDAS, and the algorithms to simulate the behavior of oil spill were studied. Finally, numerical tests were provided to illustrate the validity of the proposed model. Results indicate that the DDDAS-based simulation method can improve the prediction accuracy of oil spill incidents.
\end{abstract}

Keywords: dynamic data-driven; oil spill; emergency management; simulation; port.

\section{Introduction}

Marine crude oil transport has the characteristics of large ship capacity and high liquidity. There will be serious damages to water environment and public security once transport accidents happen. Subject to the influence of meteorology, hydrology and other factors such as oil spill diffusion and drift, the cleanup of marine oil spill accident is difficult, and the emergency management process is complicated.

Port water area has high frequency of marine oil spill incidents. The emergency management for oil spill in port water area should not only consider the features of marine oil spill, but the complex vessel traffic conditions in port water area and the influence of port production. Thus, the requirements for emergency decision and response are higher. Meanwhile, with rapid increase in crude oil transport during the recent years, many crude oil ports face increasing risk of oil spill incidents. Therefore, emergency management of oil spill in port water area receives increasing attention.

Analysis of the evolution mechanism based on the simulation and prediction of diffusion process of oil spill incidents is one of the key issues to improve science and effectiveness of emergency response. Numerous studies have been carried out. And many simulation mod- els were developed (Chen et al. 2007; Sebastião, Soares 2007; Wang et al. 2008; Guo, Wang 2009) by combination of oil spill calculation model with GIS technology and simulation modeling method to simulate and predict the oil spill diffusion process.

Marine oil spill has a complex mechanism of occurrence, development and evolution, as well as the secondary and coupled disasters. While analyzing and predicting the accidents, diffusion patterns of oil spill under the influence of complex meteorological, hydrological and environmental factors, the effect of multi-agent and stochastic factors should be considered. Due to the nonlinearity, time-variability, multivariable and uncertainty system characteristics, it is difficult to analyze and predict complex behaviors in emergency response system of oil spill incidents by using traditional analytical models. As a result, simulation technology has been widely applied. However, current simulation model mainly depends on offline historical incident data for simulation analysis. The real-time data is not used to improve the prediction accuracy of simulation model.

Dynamic Data-Driven Application System (DDDAS) is a new simulation paradigm where applications, simulations, experiments and tests become a feedback control system. In this method, simulation can dynamically as-

Corresponding author: Qingcheng Zeng

E-mail: zqcheng2000@hotmail.com 
similate data from the actual system and make response during running simulation, and the simulation results can control the actual system processing dynamically. The integration of the real-time data, simulation models, and application systems provides more efficient, reliable, and precise predictions and analyses of the characteristics and behaviors of complex systems.

Since the National Natural Science Foundation of United States first put forward DDDAS, increasing applications have been made in variety of engineering and science practices, e.g. pollutant migration (Douglas, Efendiev 2006; Douglas et al. 2004), fire simulation (Douglas et al. 2004; Mandel et al. 2005), engineering system design and control (Kim, Heller 2006; Zhao et al. 2004), ecosystem prediction (Muttil, Lee 2005), emergency medical treatment decisions (Gaylor et al. 2005), and natural disaster prediction (Parashar et al. 2004), etc.

Considering the difficulty in predicting accident developing during emergency management, a DDDAbased simulation model for oil spill emergency decision is developed to improve the accuracy of simulation and prediction of oil spill. Problems such as the method to map real-time data to simulation model, the method to recovery initial data of simulation model and the algorithms to simulate behavior of oil spill are designed.

\section{Framework of DDDAS-Based Simulation Model}

According to Ouyang et al. (2007), DDDAS is a feedback-control system that consists of the following five symbiotic features, namely, human controls, dynamic computations, application models, measurement infrastructures, and software support systems. Human controls and interacts with application models, measurement infrastructures and software support systems through computational infrastructures. And the similar concept was defined by National Natural Science Foundation of United States in 2000.

Based on the above concept, the framework of DDDAS-based simulation model for oil spill is designed as Fig. 1. In this simulation framework, historical and real-time online data are used simultaneously in simulation model to forecast and simulate oil spill diffusion. Firstly, oil spill emergency management simulation model is developed. Then simulation model is calibrated

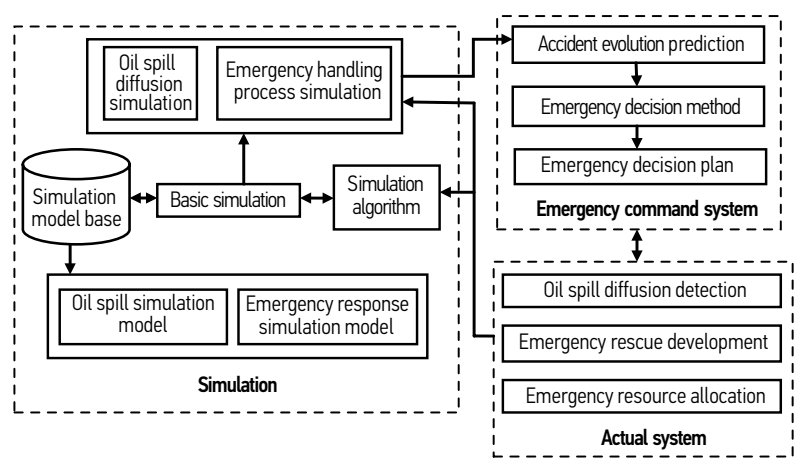

Fig. 1. Framework of DDDAS-based simulation model using massive historical data of oil spill incidents. The calibrated simulation model can be used to simulate oil spill diffusion and analyze emergency response process. When the oil spill accidents happen, the accidents data are input to the simulation model to predict the oil diffusion process. While the simulation model is running, it can dynamically assimilate real-time data from the actual system, feedback the real-time state of the accident to the simulation model. The assimilation of real-time data makes the simulation model adjust its prediction and modify the model continuously. Through the interaction of the simulation model and actual system data, the prediction accuracy of simulation model can be improved.

\section{Simulation Model}

Two problems should be solved when developing the simulation model of oil spill emergency management. The first one is abstraction of the complex spatial elements, environmental factors and oil spill diffusion behaviors into simulation model, and the second one is description of the process of emergency plans, such as the effect on accident development caused by emergency rescue, and the behaviors in accidents.

To solve the problem, non-formal object-oriented simulation modeling method is used. The objects in the emergency system are divided into entity object, information object and control object. The overall structure and logical flow of the simulation model is designed base on function analysis by non-formal object-oriented simulation modeling method. In aspect of oil spill diffusion, calculation models for oil spill behaviors such as drift, diffusion, evaporation and emulsification are developed, which can simulate the oil spill diffusion process in all directions.

The method of integrating each module in the simulation model is shown in Fig. 2. AnyLogic is the software to construct the simulation model, by which the general structure and logical flow of the simulation model are developed. ArcGIS is used as GIS platform, and the current universal S-57, S-52 standard format of electronic ocean chart is converted to ArcGIS. The integration of each module is accomplished by control module developed by Java. When the process is running, the simulation model returns to the results after accepting the simulation command of control module. Meanwhile,

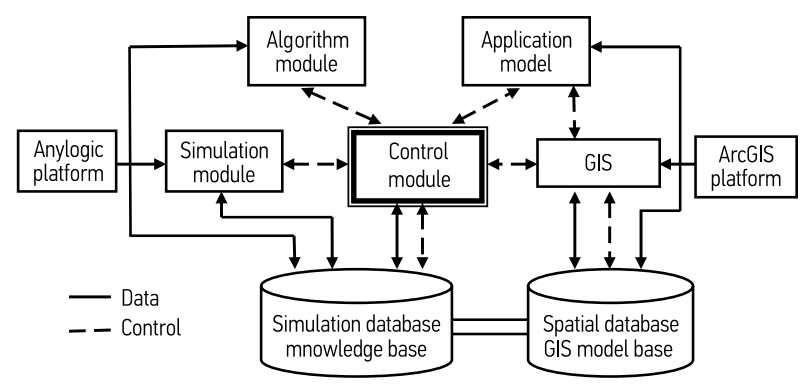

Fig. 2. Simulation model for oil spill emergency management 
the diffusion process and the demonstration of simulation data on GIS are controlled by control module. The algorithm module and application module run under the control of the control module, and exchange data with system data base on real-time.

\section{Simulation Algorithms Design}

\subsection{Algorithms for Oil Spill Diffusion}

Simulation algorithms for oil spill behaviors are needed in simulation model. When the crude oil spills in the sea, it mainly behaves as drift, diffusion, evaporation, emulsification, dissolution, dispersion, settlement and biodegradation etc. however, in the actual oil spill incidents, decision-makers mainly take measures according to the diffusion oil spill and change of oil film area. Therefore, four common behaviors, drift, diffusion, evaporation and emulsification are considered in this paper:

\section{(1) Drift model:}

The drift movement can be divided into advection process and diffusion process according to particle model equations. The advection movement mainly results from the synthesis of tidal and wind currents. The three-dimensional space position and distribution of oil spill is the comprehensive function result of various motions. According to Lagrange Equation, the displacement of single oil particle in the period of $\Delta t$ can be formulated as:

$$
\Delta \gamma_{i}=U_{i} \cdot \Delta t+W_{i} \cdot \Delta t+\gamma_{i}^{\prime},
$$

where: $\gamma_{i}$ is the position of particle $i ; U_{i}$ is the advection speed of particle $i$; $W_{i}$ is the vertical speed; and $\gamma_{i}^{\prime}$ is the random movement distance.

\section{(2) Diffusion model:}

The diffusion of oil spill in the sea includes horizontal and vertical diffusion. In recent years, the random movement method is widely used to simulate the turbulent flow diffusion process. In this method, the formulation of oil particle is regarded as the superposition of advection drift and turbulent drift. For the threedimension case, the random movement distance can be calculated by Eq. (2):

$$
\Delta a=R \cdot \sqrt{6 \cdot K_{a} \cdot \Delta t},
$$

where: $\Delta a$ is the turbulent diffusion distance in the direction $a ; R$ is a random number uniformly distributed between $[-1,1] ; K_{a}$ is the turbulent diffusion coefficient in the direction $a$; $\Delta t$ is the time step.

Moreover, oil drops in the water make random movements in vertical direction. The distance can be calculated by Eq. (3):

$$
\Delta Z=\xi \cdot \sqrt{6 \cdot K(z) \cdot \Delta t}
$$

where: $K(z)$ is vertical diffusion coefficient; $\xi$ is a random number uniformly distributed between $[-1,1]$. $K(z)$ can be represented by Eq. (4):

$$
K(z)=0.028 \cdot\left(\frac{H_{s}^{2}}{T}\right) \cdot e^{-2 \cdot k \cdot z},
$$

where: $k$ represents wave number; $T$ represents wave period; $z$ represents wave depth.

\section{(3) Evaporation model:}

By using the parametric formula proposed by Stiver and Mackay (1984), the equation for evaporation rate can be formulated as:

$$
F_{v}=\ln \left(1+B^{\prime} \cdot\left(\frac{T_{G}}{T}\right) \cdot \theta^{\prime} \cdot e\left(A^{\prime}-B^{\prime} \cdot \frac{T_{0}}{T}\right)\right) \cdot \frac{T}{B^{\prime} \cdot T_{G}},
$$

where: $F_{v}$ is evaporation rate; $T_{G}$ is the gradient of boiling point curve; $T$ is oil temperature, $T_{0}$ is original boiling temperature of oil; $\theta$ is evaporation coefficient; $A^{\prime}=6.3 ; B^{\prime}=10.3$.

\section{(4) Emulsification model:}

The equation (6) proposed by Mackay (Stiver, Mackay 1984) is used to calculate the moisture content of emulsified materials, where $Y_{w}$ is the moisture content of emulsified materials; $U_{w}$ is wind speed; $K_{A}=4.5 \cdot 10^{-6}$; $K_{A} \approx 1.25$.

$$
Y_{w}=\frac{1}{K_{B}}\left(1-e^{-K_{A} \cdot K_{B} \cdot\left(1+U_{W}\right)^{2} \cdot t}\right) .
$$

\subsection{Algorithm for Simulation Data Update}

In the DDDAS-based simulation model, one of the objectives of dynamic data driven simulations is to incorporate the real time data into simulations. As the data inputted into the simulator, algorithms are needed to assimilate real-time data and update the initial condition of simulation mode, thus modified the simulation model and improve the prediction effect. The update can be performed based on the sensor measurements as well as other large scale measurements.

The real-time data here is mainly obtained by sensor, and the initial condition refers to the condition of simulation model at the last decision-making moment, not the condition when the simulation model starts. The reason of this hypothesis is mainly for improving the operational effect of simulation model. Based on the approach proposed by Douglas and Efendiev (2006), the following updated algorithms are used.

Let $N_{s}$ denote the number of sensor; $x_{j}\left(j=1, \ldots, N_{s}\right)$ denote the position of sensor $j$; $N_{t}$ denote the number of decision-making points; $t_{k}\left(k=1, \ldots, N_{t}\right)$ denote the moment $k ; \gamma_{j}\left(t_{k}\right)$ denote the data obtained by the sensor $j$ at the moment $k$, with $M\left(\gamma, t_{k}\right)=\left\{\gamma_{j}\left(t_{k}\right), j=1, \ldots, N_{s}\right\}$. $\bar{C}_{i}^{t}(X)\left(i=1, \ldots, N_{c}\right)$ denotes the state value of simulation model at the moment $t$, which could be the state value of oil spill diffusion, as well as the state value of emulsification and evaporation etc. $N_{c}$ represents the dimensions of data in simulation model. The initial condition of simulation model is defined as:

$$
\bar{C}^{0}(X)=\sum_{i=1}^{N_{c}} \alpha_{i} \cdot \bar{C}_{i}^{0}(X) .
$$


Using the Superposition Principle, $\bar{C}_{i}^{0}(X)$ is regarded as the initial value and substituted into Eq. (8), then Eq. (9) can be obtained:

$$
\begin{aligned}
& \frac{\partial C}{\partial t}+v \cdot \nabla C-\nabla(D \cdot \nabla C)=0 \\
& \bar{C}(X, t)=\sum_{i=1}^{N_{c}} \alpha_{i} \cdot \bar{C}_{i}(X, t) .
\end{aligned}
$$

In order to calculate the difference between the actual date and simulated data, Eq. (10) is formulated:

$F(\alpha)=\sum_{j=1}^{N_{s}}\left(\sum_{i=1}^{N_{C}} \alpha_{i} \cdot \bar{C}_{i}\left(x_{j}, t\right)-\gamma_{j}(t)\right)^{2}+\sum_{i=1}^{N_{C}} k_{i} \cdot\left(\alpha_{i}-\beta_{i}\right)^{2}$,

where: $k=\left(k_{1}, k_{2}, \ldots, k_{N_{c}}\right)$ is the penalty coefficient of system information before the initial condition.

The objective of data update in simulation model is to minimize Eq. (10) by solving Eq. (11):

$$
\frac{\partial F \alpha}{\partial \alpha_{m}}=0, m=\left(1,2, \ldots, N_{C}\right) \text {. }
$$

Suppose that:

$$
\begin{aligned}
& A \cdot \alpha=R \\
& A_{m n}=\sum_{j=1}^{N_{s}} \bar{C}_{m}\left(x_{j}, t\right) \cdot \bar{C}_{n}\left(x_{j}, t\right)+\delta_{m n} \cdot k_{m},
\end{aligned}
$$

where: $R$ represents the state value after the simulation models accomplishes updating. If $m=n, \delta_{m n}=1$; otherwise, $\delta_{m n}=0$.

Therefore, the data updating of simulation models can be expressed by (12):

$$
R_{m}=\sum_{j=1}^{N_{s}} \bar{C}_{m}\left(x_{j}, t\right) \cdot \gamma(t)+k_{m} \cdot \beta_{m}
$$

These values are obtained from the sensor data, if available. At each step of the simulation, the sensor data needs to be mapped to the solution space. In addition, the sensor data is not available at some location, interpolation methods are needed to evaluate the data between the sensor points. The method proposed by Douglas et al. (2004) was used to map the sensor data to solution space and obtain the data where sensor data is not available.

\section{Numerical Experiments}

The oil spill incident that happened in Dalian port water area in 2005 is used as an example to illustrate the validity of the proposed model. The quantity of oil spill in this accident was about 240. As for there wasn't any sensor to monitor information, the data used in the analysis is dependent on the accident description material which was recorded by Maritime Department during the ac-

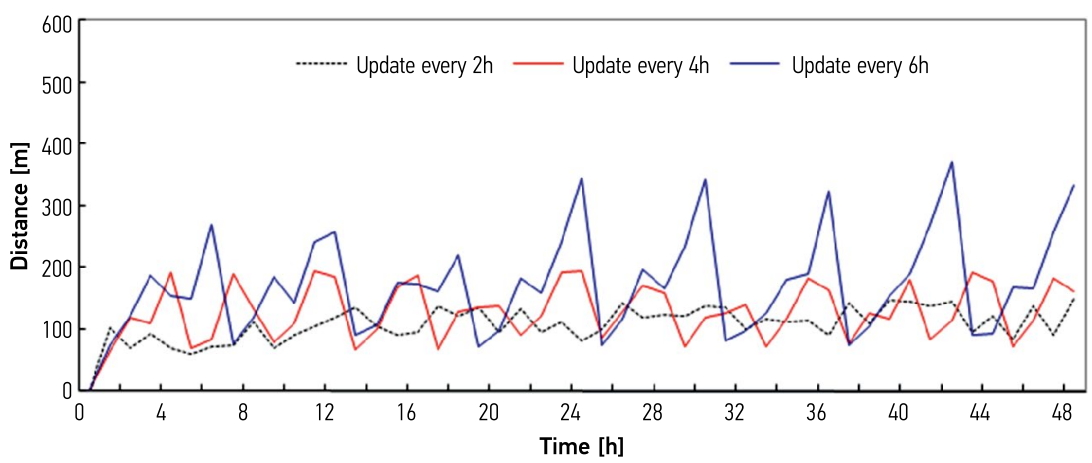

Fig. 3. The distance of central drift trajectory between DDDAS-based model and real case

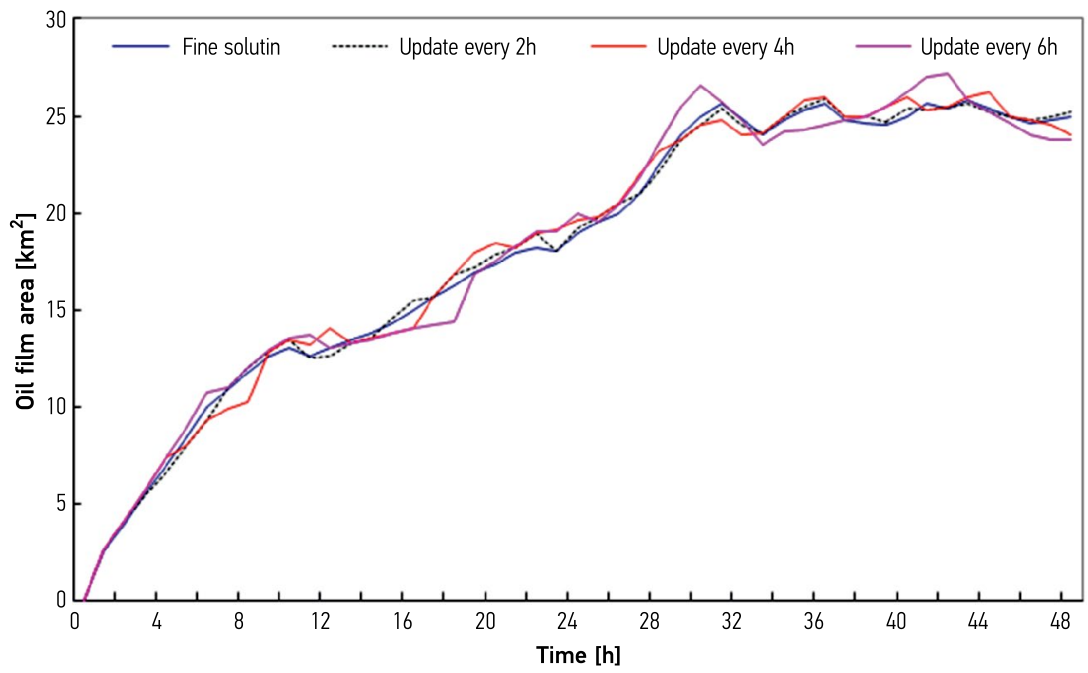

Fig. 4. Simulation results of oil film area for different updating frequency 
cident. The simulation model is updated every two, four and six hours respectively, and the real-time information includes wind speed, changes in natural conditions, and the actual position of oil spill diffusion. Considering that there are no statistics data for evaporation and emulsification behaviors, only drift and diffusion are simulated.

Firstly, the distance of central drift trajectory between DDDAS-based model and real case is calculated. In DDDAS-based model, the simulation model is updated every two, four and six hours. And the drift of 48 hours after oil spill accident is simulated. Results are shown in Fig. 3. From the results in Fig. 3, we can find that the difference between simulated model and real case increases with the increasing of updating time interval. This indicates that the prediction accuracy of simulation model improves with the increasing of data updating frequency.

Moreover, similar results are shown in the results of the oil film area is simulation (Fig. 4). With the increasing of updating frequency, the prediction accuracy of oil film area improves.

Results indicate that simulation model with high updating frequency is more closed to the actual diffusion process compared to those with low updating frequency, which indicates the validity of DDDAS-based model. DDDAS model can absorb real-time data and adjust the prediction results in time, thus to the increase of oil spill simulation accuracy.

\section{Conclusions}

1. In this paper, a DDDAS-based simulation model for oil spill emergency decision was developed. By assimilating real-time data, simulation model can update its initial condition and modify its parameters. Results indicate that the proposed could predict oil spill accident more precisely compared to general simulation models.

2. In practical applications, real-time data can be obtained through the way to install sensors on port water area. Recently sensing technology developed rapidly, which provides better prospect for the application of DDDAS method. Without sensors, real-time data can be acquired by present observation technology, which can also increase the model accuracy to some extent.

3. In the DDDAS-based model, one of the key problems is how to receive data dynamically from actual system and make response simultaneously. Further studies are required to focus on the improvement of two aspects: one is the method to mapping real-time data to simulation model, and the other is the method to recovery initial data for DDDAS. In this paper, there are no real-time sensor data in numerical experiments; further experiments with real-time sensor data are needed in future studies.

\section{Acknowledgements}

This work is supported by National Natural Science Foundation of China (No. 71001012), Foundation of Educational Office of Liaoning Province (No. 20090451261) and Program for New Century Excellent Talents in University.

\section{References}

Chen, H.-Z.; Li, D.-M.; Li, X. 2007. Mathematical modeling of oil spill on the sea and application of the modeling in Daya Bay, Journal of Hydrodynamics, Ser. B 19(3): 282-291. http://dx.doi.org/10.1016/S1001-6058(07)60060-2

Douglas, C. C.; Efendiev, Y. 2006. A dynamic data-driven application simulation framework for contaminant transport problems, Computers \& Mathematics with Applications 51(11): 1633-1646.

http://dx.doi.org/10.1016/j.camwa.2006.05.003

Douglas, C. C.; Shannon, C. E.; Efendiev, Y.; Ewing, R.; Ginting, V.; Lazarov, R.; Cole, M. J.; Jones, G., Johnson, C. R.; Simpson, J. 2004. A note on data-driven contaminant simulation, Lecturer Notes in Computation Science 3038: 701-708. http://dx.doi.org/10.1007/978-3-540-24688-6_91

Gaylor, M.; Seltzer, M.; Moulton, S.; Freedman, J. 2005. A dynamic, data-driven, decision support system for emergency medical services, Lecture Notes in Computation Science 3515: 703-711. http://dx.doi.org/10.1007/11428848_91

Guo, W. J.; Wang, Y. X. 2009. A numerical oil spill model based on a hybrid method, Marine Pollution Bulletin 58(5): 726734. http://dx.doi.org/10.1016/j.marpolbul.2008.12.015

Kim, S.; Heller, M. 2006. Emerging cyberinfrastructure: challenges for the chemical process control community, Computers \& Chemical Engineering 30(10-12): 1497-1501. http://dx.doi.org/10.1016/j.compchemeng.2006.04.007

Mandel, J.; Bennethum, L. S.; Chen, M.; Coen, J. L.; Douglas, C. C.; Franca, L. P.; Johns, C. J.; Kim, M.; Knyazev, A. W.; Kremens, R.; Kulkarni, V.; Qin, G.; Vodacek, A.; Wu, J.; Zhao, W.; Zornes, A. 2005. Towards a dynamic data driven application system for wildfire simulation, Lecturer Notes in Computation Science 3515: 632-639.

http://dx.doi.org/10.1007/11428848_82

Muttil, N.; Lee, J. H. W. 2005. Genetic programming for analysis and real-time prediction of coastal algal blooms, Ecology Modelling 189(3-4): 363-376.

http://dx.doi.org/10.1016/j.ecolmodel.2005.03.018

Ouyang, Y.; Zhang, J. E.; Luo, S. M. 2007. Dynamic data driven application system: recent development and future perspective, Ecological Modelling 204(1-2): 1-8. http://dx.doi.org/10.1016/j.ecolmodel.2006.12.010

Parashar, M.; Klie, H.; Catalyurek, U.; Kurc, T.; Bangerth, W.; Matossian, V.; Saltz, J.; Wheeler, M. F. 2004. Application of grid-enabled technologies for solving optimization problems in data-driven reservoir studies, Lecture Notes in Computer Science 3038: 805-812.

http://dx.doi.org/10.1007/978-3-540-24688-6_104

Sebastião, P.; Soares, C. G. 2007. Uncertainty in predictions of oil spill trajectories in open sea, Ocean Engineering 34(3-4): 576-584. http://dx.doi.org/10.1016/j.oceaneng.2006.01.014

Stiver, W.; Mackay, D. 1984. Evaporation rate of spills of hydrocarbons and petroleum mixtures, Environmental Science \& Technology 18(11): 834-840. http://dx.doi.org/10.1021/es00129a006

Wang, S.-D.; Shen, Y.-M.; Guo, Y.-K.; Tang, J. 2008. Three-dimensional numerical simulation for transport of oil spills in seas, Ocean Engineering 35(5-6): 503-510. http://dx.doi.org/10.1016/j.oceaneng.2007.12.001

Zhao, H.; Knight, D.; Taskinoglu, E.; Jovanovic, V. 2004. Data driven design optimization methodology development and application, Lecturer Notes in Computation Science 3038: 748-755. http://dx.doi.org/10.1007/978-3-540-24688-6_97 